\title{
Metabolomic Study of Collagen-Induced Arthritis in Rats and the Interventional Effects of Huang-Lian-Jie-Du-Tang, a Traditional Chinese Medicine
}

\author{
Rongcai Yue, ${ }^{1}$ Ling Zhao, ${ }^{1,2}$ Yaohua Hu, ${ }^{1}$ Peng Jiang, ${ }^{1}$ Shuping Wang, ${ }^{1}$ Li Xiang, \\ Wencong Liu, ${ }^{2}$ Lei Shan, ${ }^{1}$ Weidong Zhang, ${ }^{1,3}$ and Runhui Liu ${ }^{1}$ \\ ${ }^{1}$ School of Pharmacy, Second Military Medical University, 325 Guo-He Road, Shanghai 200433, China \\ ${ }^{2}$ College of Traditional Chinese Medicine, Jilin Agricultural University, Changchun 130118, China \\ ${ }^{3}$ School of Pharmacy, Shanghai Jiao Tong University, Shanghai 200030, China
}

Correspondence should be addressed to Weidong Zhang; wdzhangy@hotmail.com and Runhui Liu; lyliurh@126.com

Received 18 September 2012; Revised 28 January 2013; Accepted 28 January 2013

Academic Editor: Wei Jia

Copyright (C) 2013 Rongcai Yue et al. This is an open access article distributed under the Creative Commons Attribution License, which permits unrestricted use, distribution, and reproduction in any medium, provided the original work is properly cited.

\begin{abstract}
Huang-Lian-Jie-Du-Tang (HLJDT) is a traditional Chinese medicine (TCM) with anti-inflammatory activity. The present study used a metabolomic approach based on LC-Q-TOF-MS to profile rheumatoid-arthritis- (RA-) related metabolic changes and to investigate the interventional mechanisms of HLJDT in collagen-induced arthritis rats. Forty male Wistar rats were randomly divided into five groups: (1) a model group, (2) a normal control group, (3) a dexamethasone group, (4) a HLJDT group, and (5) a group that received 13 components of HLJDT. Plasma samples were collected 8,15 , and 22 days after the rats were injected with bovine type II collagen. By combining variable importance in the projection values with partial least squares discriminant analysis, 18 potential biomarkers were identified in the plasma samples. The biomarkers were primarily involved in glycerophospholipid metabolism, fatty acid metabolism, tryptophan metabolism, linoleic acid metabolism, phenylalanine metabolism, purine metabolism, arachidonic acid metabolism, and bile acid biosynthesis. Using the potential biomarkers as a screening index, the results suggest that HLJDT can potentially reverse the process of RA by partially regulating fatty acid oxidation and arachidonic acid metabolism. This study demonstrates that a metabolomic strategy is useful for identifying potential RA biomarkers and investigating the underlying mechanisms of a TCM in RA treatment.
\end{abstract}

\section{Introduction}

Rheumatoid arthritis (RA) is an autoimmune disease characterized by persistent synovitis, systemic inflammation, and autoantibodies $[1,2]$. RA primarily affects the small diarthrodial joints of the hands and feet, causing swelling and pain. If left untreated, RA may result in deformity. Several studies have investigated the pathogenetic mechanisms of RA, particularly collagen-induced arthritis (CIA) in humans and in animal models [3-5]. Although some important biomarkers and novel therapeutic methods have been identified and developed for RA diagnosis and treatment [68], the occurrence of RA-induced deformity remains high. Moreover, current RA treatment medications are limited by several well-characterized clinical side effects, such as hepatotoxicity $[9,10]$, gastrointestinal effects [11], and cardiotoxic effects [12]. Therefore, further investigation of the biological processes related to RA, as well as its clinical diagnosis and treatment, is needed to understand its disease mechanism, identify new biomarkers, and explore new anti-RA drugs.

Metabolomics focuses on the comprehensive measurement of all small molecular weight compounds, including endogenous and exogenous species, which are present in a biological system. Furthermore, it provides a functional readout of abnormal, disease-related physiological states in the human body and may provide new insights into the global effects of disease related to metabolic pathways [13]. In recent years, metabolomics has been used to identify disease-related 
biomarkers and shows significant potential for the early diagnosis, therapeutic monitoring and pathogenic understanding of many diseases, including RA [14-16]. Among the analytical techniques used in metabolomic research, LC-MS is recognized as one of the most selective, sensitive, and reproducible methods [17] due to its enhanced reproducibility of retention times [18]. This reproducibility is especially important for large-scale, untargeted metabolic profiling.

Huang-Lian-Jie-Du-Tang is an aqueous extract that consists of four herbal materials: Rhizoma Coptidis, Radix Scutellariae, Cortex Phellodendri, and Fructus Gardeniae. In our previous study, LC-DAD and LC-ESI-MS methods were developed and validated for the chromatographic fingerprinting and quantitative analysis of HLJDT [19]. Furthermore, HLJDT's potentially active components were identified in a plasma-based pharmacochemical study [20]. In addition, the anti-inflammatory activities, component herbs and active components of HLJDT were also investigated [21, 22]. However, a proper approach for evaluating the holistic efficacy of such a multicomponent medicine is urgently needed. In the present study, we used a metabolomic approach to investigate the biochemical abnormalities associated with RA and to assess the therapeutic effects of HLJDT and its components in CIA rats.

\section{Experimental Methods}

2.1. Reagents and Materials. HPLC-grade methanol and acetonitrile were purchased from J.T. Baker (NJ, USA). Ultrapure water (18.2 M $\Omega$ ) was prepared with a Milli-Q water purification system (Millipore, MA, USA). The following HLJDT components were purchased from the National Institute for the Control of Pharmaceutical and Biological Products (Beijing, China): geniposide, coptisine, phellodendrine, jatrorrhizine, magnoflorine, palmatine, berberine, baicalin, chlorogenic acid, crocin, wogonoside, baicalein, and wogonin. The following standard metabolites were obtained from Sigma-Aldrich (St. Louis, MO, USA): choline, carnitine, Lphenylalanine, arachidonic acid, hippuric acid, uric acid, allantoin, 5-hydroxy tryptophan, and L-tryptophan.

Rhizoma Coptidis (Rhizoma of Coptis chinensis Franch.), Radix Scutellariae (Radix of Scutellaria baicalensis Georgi.), Cortex Phellodendri (Cortex of Phellodendron chinense Schneid.), and Fructus Gardeniae (Fructus of Gardenia jasminoides Ellis.) were purchased from Bozhou (Anhui province, China) and were authenticated by Professor HanMing Zhang (Second Military Medical University, Shanghai, China). Voucher specimens of Rhizoma coptidis, Radix scutellariae, Cortex phellodendri, and Fructus gardeniae were stored at the Second Military Medical University, Shanghai, China (no. HL20070523, HQ20070523, HB20070523, and ZZ20070523, resp.). HLJDT extract was prepared from the four medicinal herbs (Rhizoma Coptidis, Radix Scutellariae, Cortex Phellodendri, and Fructus Gardeniae in a 3:2:2:3 ratio) as previously described in $[19,20]$. All TCM mixtures were maintained under careful quality control to ensure their identification throughout the experiments.
2.2. Animals. Adult male Wistar rats (140-160 g) were purchased from the SLAC Laboratory Animal Co. (Shanghai, China). Rats were kept in SPF-grade Experimental Animal Houses (the Second Military Medical University, Shanghai) with free access to food and water under standard temperature conditions $\left(22^{\circ} \mathrm{C}\right)$ and a $12 \mathrm{~h}$ light/dark cycle. The animal experiments were conducted in strict accordance to the National Institutes of Health's Guide to the Care and Use of Laboratory Animals. The animal experiments were approved by the local institutional review board at the authors' affiliated institutions.

2.3. CIA Model and Drug Administration. Type II collagen (Chondrex, Redmond, WA, USA) was emulsified with incomplete Freund's adjuvant at a 1:1 ratio. Rats were intradermally injected with $2 \mathrm{mg} / \mathrm{kg}$ of collagen-IFA suspension at the base of the tail (day 0). A boost injection with $1 \mathrm{mg} / \mathrm{kg}$ of the collagen-IFA suspension was given on day 7 in the same manner.

Forty rats were randomly divided into 5 groups of 8 rats each: (1) rats without CIA immunization (normal control group, NG), (2) rats with CIA immunization (CIA model group, MG), (3) CIA rats treated with $270 \mathrm{mg} / \mathrm{kg}$ of HLJDT (HLJDT group, HG), (4) CIA rats treated with the 13 main components of HLJDT (components group, CG), and (5) rats treated with $0.05 \mathrm{mg} / \mathrm{kg}$ of dexamethasone (Sine Phama Lab Co., Ltd., Shanghai, China) (positive control group, DG). A dry powder of HLJDT was dissolved in $0.5 \%$ carboxymethyl cellulose sodium (CMC-Na), stirred at $37^{\circ} \mathrm{C}$ for $1 \mathrm{~h}$ and administered orally to the CIA rats. This $270 \mathrm{mg} / \mathrm{kg}$ dose was explored in the animal experiment and is considered within the MTD $(2 \mathrm{~g} / \mathrm{kg})$ for oral administration. Based on the quantitative analysis of HLJDT [19] and a dosage of $270 \mathrm{mg} / \mathrm{kg}$ of HLJDT, 13 main components were identified: $5.67 \mathrm{mg} / \mathrm{kg}$ of geniposide, $0.24 \mathrm{mg} / \mathrm{kg}$ of coptisine, $0.35 \mathrm{mg} / \mathrm{kg}$ of phellodendrine, $1.2 \mathrm{mg} / \mathrm{kg}$ of jatrorrhizine, $1.31 \mathrm{mg} / \mathrm{kg}$ of magnoflorine, $2.07 \mathrm{mg} / \mathrm{kg}$ of palmatine, $12.97 \mathrm{mg} / \mathrm{kg}$ of berberine, $10.55 \mathrm{mg} / \mathrm{kg}$ of baicalin, $1.05 \mathrm{mg} / \mathrm{kg}$ of chlorogenic acid, $0.39 \mathrm{mg} / \mathrm{kg}$ of crocin, $2.39 \mathrm{mg} / \mathrm{kg}$ of wogonoside, $1.45 \mathrm{mg} / \mathrm{kg}$ of baicalein, and $0.88 \mathrm{mg} / \mathrm{kg}$ of wogonin. The components were mixed, dissolved in $0.5 \%$ CMC-Na solution, and administered intragastrically to the CIA rats. The NG and $\mathrm{MG}$ rats received oral administrations of an equal volume of $0.5 \%$ CMC-Na aqueous solution. All of the drug treatments were administered daily from day 0 to 28 .

2.4. Assessment of Arthritis in Rats. After the second immunization, the rats were checked for the development of arthritis based on the extent of edema and/or erythema in their paws. The incidence and severity of arthritis were evaluated by observing changes in their arthritis scores every 2 days, measuring hind paw volumes every 4 days and measuring body weight every 3 days (only when arthritic signs were present). The observed severity of the arthritis was assessed by a semiqualitative score as follows: 0 , normal, with no macroscopic signs of arthritis or swelling; 1, mild but distinct redness and swelling of the ankle or apparent redness and swelling of the individual digits, regardless of the number 
of affected digits; 2, moderate redness and swelling of the ankle; 3 , redness and swelling of the entire paw, including the digits; and 4, maximally inflamed limb with the involvement of multiple joints. In these studies, the maximum score was 8 , which represents the sum of the scores of both hind paws in each animal. The hind paw volumes were measured with a plethysmometer (7140UGO, Basile, Comerio, Italy) and were recorded as the mean volume displacement of both hind paws in each rat. A precision balance (Sartorius AG, Goettingen, Germany) was used to monitor changes in body weight.

\subsection{Lipid Peroxide Assay and Antioxidant Enzyme Activity} Assays. The plasma samples were obtained by centrifuging blood samples for $10 \mathrm{~min}$ at $3500 \mathrm{rpm}$ and $4^{\circ} \mathrm{C}$. The supernatant was used in the subsequent bioassays. Malondialdehyde (MDA), superoxide dismutase (SOD), and glutathione peroxidase (GSH-Px) assays were performed using commercially available kits according to their manufacturer's instructions (Jiancheng Bioengineering Institute, Nanjing, China). Briefly, the lipid peroxide content was determined by measuring the concentration of thiobarbituric-acid- (TBA-) reactive substances. The TBA-reactive content was expressed in terms of MDA content using 1,1,3,3-tetraethoxypropane as a standard. The absorbance was measured at $532 \mathrm{~nm}$ and the values were expressed as nmol of MDA per $\mathrm{mg}$ of protein. The SOD assay was based on SOD's inhibitory effects on the spontaneous autoxidation of 6-hydroxydopamine. One IU of SOD is required to inhibit the initial rate of 6hydroxydopamine autoxidation by $50 \%$. The GSH-Px activity assay is based on measurements of decreasing absorbance at $340 \mathrm{~nm}$ due to the consumption of NADPH.

2.6. Sample Preparation. The plasma samples were collected from the NG, MG, DG, HG, and CG on days 8,15 , and 22 . The samples were stored at $-80^{\circ} \mathrm{C}$ prior to analysis. One-hundredmicroliter aliquots of plasma were diluted with $300 \mu \mathrm{L}$ of methanol. After vortex-mixing the solution for $1 \mathrm{~min}$ and centrifuging it at $12000 \mathrm{rpm}$ for $10 \mathrm{~min}$, the supernatant was transferred to autosampler vials. A quality control (QC) sample was prepared by mixing $20 \mu \mathrm{L}$ aliquots from each group with plasma and handled in the same manner described above. The QC sample was used for monitoring the stability of sequence analysis and was continuously analyzed 6 times to validate the repeatability of the equipment.

2.7. LC-Q-TOF-MS Conditions. LC-Q-TOF-MS analysis was performed on an Agilent-1290 LC system (Agilent Technologies, Palo Alto, CA, USA) coupled with an electrospray ionization (ESI) source and an Agilent-6530 Q-TOF mass spectrometer. Chromatographic separation was performed on a Zorbax SB-C18 column $(1.8 \mu \mathrm{m}, 2.1 \mathrm{~mm} \times 150 \mathrm{~mm}$, Agilent) with the column temperature set at $40^{\circ} \mathrm{C}$. Ultrapure water with $0.1 \%$ formic acid (A) and acetonitrile (B) was used in the mobile phase according to the following gradient program: $0-2 \mathrm{~min}, 5 \% \mathrm{~B}$; and 2-5 $\mathrm{min}, 5-50 \% \mathrm{~B}$; and 5-6 min, $50 \% \mathrm{~B} ; 6-17 \mathrm{~min}, 50-95 \% \mathrm{~B}$; followed by a $5-\mathrm{min}$ reequilibration step. The mobile phase flow rate was $0.3 \mathrm{~mL} / \mathrm{min}$, and the sample injection volume was $4 \mu \mathrm{L}$.
Positive and negative ion modes were used in mass detection. The source parameters were set as follows: drying gas flow rate, $11 \mathrm{~L} / \mathrm{min}$; gas temperature, $350^{\circ} \mathrm{C}$; pressure of nebulizer gas, 45 psig; Vcap, $4000 \mathrm{~V}$ in positive mode and $3000 \mathrm{~V}$ in negative mode; fragmentor, $120 \mathrm{~V}$; skimmer, $45 \mathrm{~V}$; and scan range, $m / z$ 50-1000. The MS/MS analysis was acquired in targeted MS/MS mode with the collision energy ranging from $10 \mathrm{~V}$ to $40 \mathrm{~V}$.

2.8. Data Processing, Multivariate Data Analysis and Biomarker Identification. The MS spectra were processed using Agilent's Mass Hunter Qualitative Analysis Software (Version B.03.01, Agilent Technologies, USA) for peak detection. A list of detected peak intensities was generated using the retention time $m / z$ data pairs as identifiers. The resultant normalized peak intensities formed a single matrix with retention time $\mathrm{m} / \mathrm{z}$ pairs for each file in the data set. All of the processed data were normalized and scaled for each chromatogram prior to multivariate statistical analysis. Integrated raw mass spectrometric data were processed using Agilent's Mass Profiler Software (Version B.02.00, Agilent Technologies, USA). The intensity of each ion was normalized with respect to the total ion count to generate a data matrix consisting of the retention time, the $m / z$ value, and the normalized peak area. The ion intensity of each peak was normalized to 10,000 and to the sum of its peak intensities within the sample. The processed data were exported and further processed by PCA and PLS-DA using the SIMCA-P software package (Version 11, Umetrics AB, Umeå, Sweden). The data were processed by unit variance scaling and were mean-centered using the SIMCA-P software. Model quality was evaluated based on the relevant values of $R^{2}$ and $Q^{2}$. Potential markers of interest were extracted from the values of variable importance in the projections (VIP > 1), which were constructed from PLSDA analysis. $P$ values were obtained from Student's $t$-test $(P<0.05)$. The exact molecular mass data from redundant $\mathrm{m} / z$ peaks, which correspond to the formation of different parent and product ions, were used to confirm the molecular mass of the metabolites. MS/MS data analysis highlighted neutral losses or product ions, which are characteristic of metabolite groups and can be used to discriminate between database hits. The identities of specific metabolites were confirmed by comparing their mass spectra and chromatographic retention times to commercially available reference standards. The metabolites were also identified at the Scripps Center for Metabolomics and Mass Spectrometry (METLIN). The biochemical reactions associated with these metabolites were obtained from the Kyoto Encyclopedia of Genes and Genomes (KEGG) and the Human Metabolome Database (HMDB). The fold changes were calculated as Fold $=\log _{2}$ (average peak intensity of group A/average peak intensity of group B).

2.9. Statistical Analysis. All quantitative data were expressed as the mean \pm SD as indicated. The comparisonsbetween the two groups were analyzed by an unpaired Student $t$-test and multiple comparisons were analyzed by one-way analysis of 
TABLE 1: Effects of HLJDT and its components on MDA levels and antioxidant enzymes' activities on day 22.

\begin{tabular}{lccc}
\hline Groups & MDA $(\mathrm{nmol} / \mathrm{mL})$ & $\mathrm{SOD}(\mathrm{U} / \mathrm{mL})^{\mathrm{a}}$ & $\mathrm{GSH}-\mathrm{Px}(\mathrm{U} / \mathrm{mL})^{\mathrm{b}}$ \\
\hline Normal control & $2.71 \pm 0.34$ & $4.56 \pm 0.24$ & $7.72 \pm 0.71$ \\
Model control & $4.98 \pm 0.53^{\# \#}$ & $2.95 \pm 0.22^{\# \#}$ & $4.97 \pm 1.56^{\#}$ \\
Dexamethasone & $2.62 \pm 0.28^{* *}$ & $4.23 \pm 0.33^{* *}$ & $7.69 \pm 1.38^{*}$ \\
HLJDT & $2.99 \pm 0.38^{* *}$ & $4.74 \pm 0.29^{* *}$ & $7.30 \pm 1.13^{*}$ \\
Components of HLJDT & $2.91 \pm 0.42^{* *}$ & $5.27 \pm 0.22^{* *}$ & $6.82 \pm 0.91^{*}$ \\
\hline
\end{tabular}

SOD: superoxide dismutase; GSH-Px: glutathione peroxidase. ${ }^{*} P<0.05,{ }^{* *} P<0.01,{ }^{\#} P<0.05$, and ${ }^{\# \#} P<0.01\left({ }^{\#}\right.$ : compared with normal control group; ${ }^{*}$ : compared with model control group).

${ }^{a}$ One unit of SOD activity is defined as amount of SOD when SOD inhibition ratio reaches $50 \%$ in $1 \mathrm{~mL}$ reaction solution.

${ }^{\mathrm{b}}$ One unit of GSH-Px activity is defined as amount of enzyme required to degrade $1 \mu \mathrm{mol} / \mathrm{L}$ of GSH per min subtracting nonenzymatic reaction at $37^{\circ} \mathrm{C}$.

variance (ANOVA) followed by Tukey's HSD post hoc test. Statistical significance was established as $P<0.05$.

\section{Results and Discussion}

3.1. Assessment of the CIA Model. Immunization with bovine type II collagen (coadministered with incomplete Freund's adjuvant) started producing severe arthritis 10 days after primary immunization and reached a peak on day 22 in the model group (Figure 1(a)). The decrease of arthritis in CIA rats that were treated with HLJDT and its components was further examined. Compared to the model group, swollen paws were significantly reduced in the dexamethasone, HLJDT, and component groups: $(P<0.05)$ (Figure 1(b)). Furthermore, after type II collagen immunization, the arthritis scores of CIA rats in the dexamethasone, HLJDT and components groups were significantly lower than those in the model group on days 16-22 $(P<0.01, P<0.05$, and $P<0.05$ versus model group, resp.) (Figure 1(c)).

3.2. Effects of HLJDT and Its Components on Lipid Peroxide and Antioxidant Enzyme Activities. Immunization with bovine type II collagen caused a significant decrease in the activities of SOD $(2.95 \pm 0.22$ versus $4.56 \pm 0.24, P<0.01)$ and GSH-Px $(4.97 \pm 1.26$ versus $7.72 \pm 0.71, P<0.05)$ and a significant increase in MDA levels (4.98 \pm 0.53 versus $2.71 \pm$ $0.34, P<0.01)$ in comparison to the normal group on day 22 (Table 1). Compared to the test groups, the administration of HLJDT and its components caused an increase in SOD and GSH-Px activity and a decrease in MDA levels in the model control group. These findings indicate that HLJDT and its components possess potent antioxidant activities in CIA rats.

3.3. Assessment of the Repeatability and Stability of the LC-QTOF-MS Method. The repeatability and stability of the LCQ-TOF-MS method were validated by analyzing 6 injections of identical QC samples that were prepared according to the same protocol. The relative standard deviations of the peak retention times and areas were less than $1.0 \%$ and $5.0 \%$, respectively. Thus, the precision and repeatability of the proposed method were satisfactory for metabolomic analysis.

Fingerprints of the plasma samples were acquired in positive and negative modes. After comparing our results between both nodes, we observed higher noise, fewer peaks, and a matrix effect in the negative mode, whereas the total ionic chromatogram (TIC) of the positive mode was more suitable for analysis (Figure 2). Moreover, most of the metabolites that were detected in the plasma samples were less polar than those observed in the urine samples described in our previous study [23].

3.4. Multivariate Statistical Analysis and Potential Biomarker Identification. Ions were generated in the LC-Q-TOF-MS analysis. PLS-DA, a supervised method, is frequently used to classify groups that show metabolic differences and to extract potential biomarkers. After PLS-DA processing, the CIA model group was clearly separated from the normal control group on day 22 (Figure 3). Variables were also generated based on the values of variable importance in the projection (VIP > 1). Then, by combining Student's $t$-test with the selected variables, distinct metabolites were identified $(P<$ 0.05 ) and selected for further study.

The three steps to identify these biomarkers were as follows. First, the $\mathrm{MS}^{2}$ spectrum of significantly different metabolic ions was obtained using a targeted MS/MS mode. Next, several online databases, such as METLIN (http://metlin.scripps.edu/), HMDB (http://www.hmdb.ca/), and KEGG (http://www.kegg.jp/), were used for initial determination of the markers. Finally, the metabolites were compared to the standard $\mathrm{MS}^{2}$ spectrum (see Figure 4 for an example using carnitine at $\mathrm{m} / z 162$ to illustrate the identification process).

Following the identification process, 18 unique metabolites were identified (Table 2), including 11 identified in the positive mode and 7 identified in the negative mode. Three of the metabolites (L-phenylalanine, allantoin, and indoxyl sulfate) were repeatedly detected in the urine samples described in our previous study. Furthermore, 13 metabolites were upregulated, and 5 metabolites were downregulated in the model group compared to the normal control group (Figure 5). These metabolites were mainly associated with glycerophospholipid metabolism, fatty acid metabolism, tryptophan metabolism, linoleic acid metabolism, phenylalanine metabolism, purine metabolism, arachidonic acid metabolism, and bile acid biosynthesis pathways and may indicate the potential efficacy of the medication in RA.

Overproduction of oxidants leads to oxidative tissue damage at the molecular level. A growing number of reports 


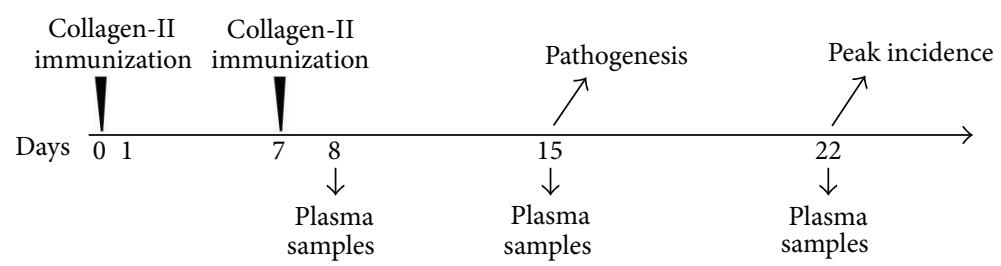

Drug administered orally once a day for 28 days

(a)

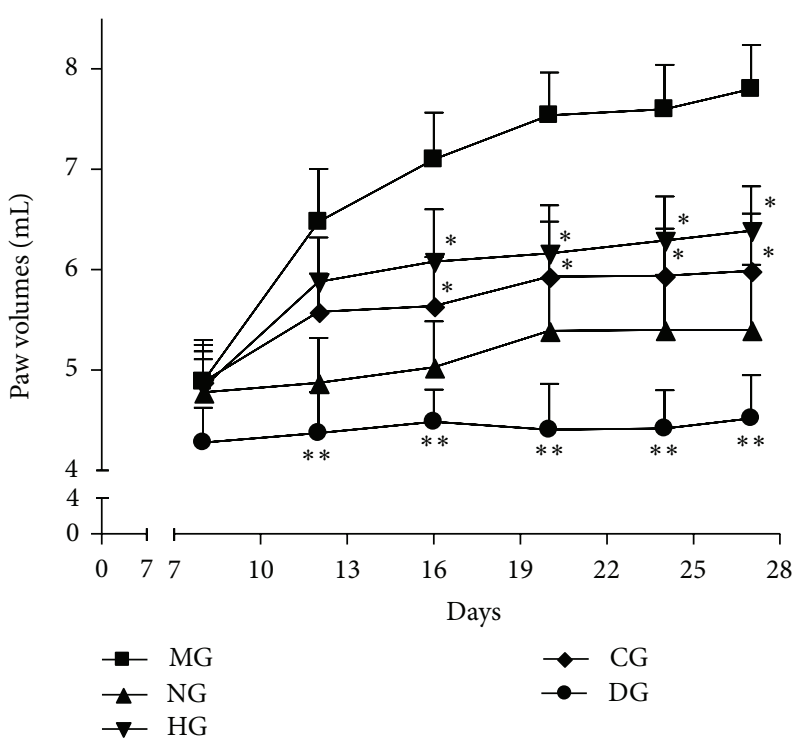

(b)

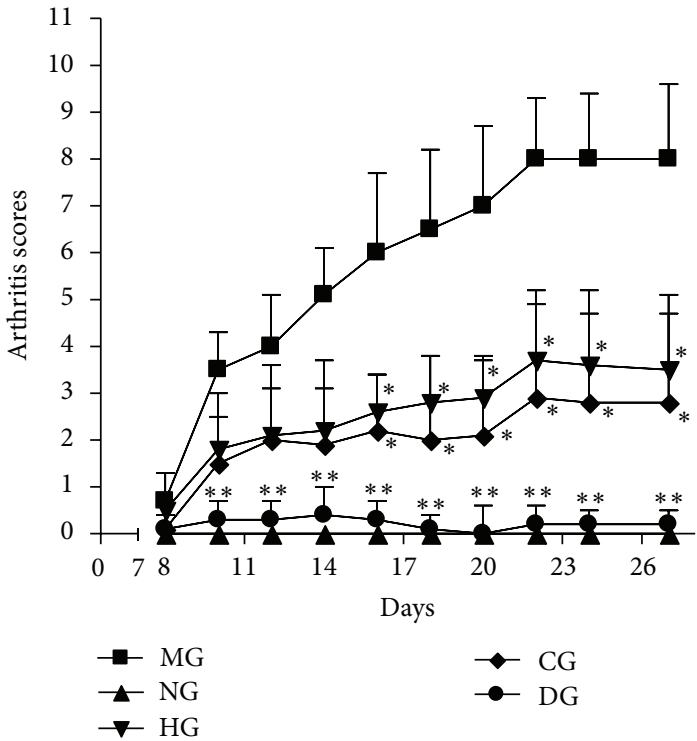

(c)

FIgURE 1: (a) Time schedule for CIA immunization, pathogenesis, peak incidence, drug administration, and sample collection for examinations. Wistar rats were immunized with bovine type II collagen with incomplete Freund's adjuvant and randomly divided into normal control, model, dexamethasone $(0.05 \mathrm{mg} / \mathrm{kg})$, HLJDT $(270 \mathrm{mg} / \mathrm{kg})$, and its 13 -component groups on the day of arthritis onset (day $0, n=8)$. (b) Hind paw volumes of each rat were evaluated every 4 days. Oral treatment of CIA rats with dexamethasone $(0.05 \mathrm{mg} / \mathrm{kg}), \mathrm{HLJDT}(270 \mathrm{mg} / \mathrm{kg})$, and its components daily significantly ameliorated the severity and development of arthritis from day $16(P<0.05$ versus model control). (c) Arthritis was scored every 2 days. Oral treatment of CIA rats with dexamethasone $(0.05 \mathrm{mg} / \mathrm{kg})$, HLJDT (270 mg/kg), and its components daily significantly reduced arthritis from day $16(P<0.01, P<0.05$, and $P<0.05$ versus model group, resp.). NG, normal control group; MG, model group; DG, dexamethasone group; HG, HLJDT group; CG, components group.

have provided evidence that implicates oxidative injury as a major pathogenic mechanism in RA [24-27]. Therefore, protecting joints from oxidative injury may provide a useful therapeutic potential for RA prevention and treatment [28, 29]. Biomarkers related to glycerophospholipid metabolism (e.g., choline and glycerophosphocholine) and fatty acid metabolism (e.g., carnitine, acetylcarnitine, palmitoyl-Lcarnitine and palmitic acid methyl ester) were all upregulated in CIA rats (except for carnitine), indicating that RA caused increased lipid catabolism. Uric acid and allantoin also contributed to oxidative injury in vivo [30-32]. Uric acid protected the DNA against free-radical damage [33, 34], while allantoin, which was detected and measured in biological fluids and tissues, was produced after uric acid oxidation. Therefore, the downregulation of uric acid and the upregulation of allantoin observed in the model group indicate that oxidative reactions led to serious damage in CIA rats. This observation also demonstrates that urate plays the role of a natural antioxidant related to purine metabolism in vivo. Carnitine is required for the transport of long chain fatty acids and acyl coenzyme A derivatives across the inner mitochondrial membrane. Several reports have shown that carnitine has protective effects against oxidative damage [35]. Carnitine not only participates in the metabolism of reactive oxygen species [14] but also plays a role in fatty acid energy metabolism [36, 37]. Other studies have shown that plasma carnitine levels were significantly lower in RA patients than in a control group [38], whereas long chain acylcarnitine levels were higher in RA patients [39]. These results are consistent with the ionic response trends that were observed in the present study. Notably, bile acid promotes the digestion and absorption of fatty acids and has an ameliorating effect on RA [40]. Bile acid biosynthesis (e.g., glycocholic acid and deoxycholic acid) was downregulated in CIA rats, indicating a disruption in fatty acid metabolism. Phenylalanine and tryptophan metabolism was discussed in our previous study 
TABle 2: Potential biomarkers in response to RA and their metabolic pathways.

\begin{tabular}{|c|c|c|c|c|c|c|c|c|}
\hline Mode 1 & Number & $t_{R} / \min$ & $m / z$ & Formula & Identification & Fold $^{\mathrm{a}}$ & $P$ value $^{\mathrm{a}}$ & Related pathway \\
\hline \multirow{11}{*}{$\mathrm{ESI}(+)$} & 1 & 1.16 & 104.1076 & $\mathrm{C}_{5} \mathrm{H}_{14} \mathrm{NO}$ & Choline $^{\mathrm{b}}$ & 26.11 & 0.000 & Glycerophospholipid metabolism \\
\hline & 2 & 1.19 & 162.1129 & $\mathrm{C}_{7} \mathrm{H}_{15} \mathrm{NO}_{3}$ & Carnitine $^{\mathrm{b}}$ & -0.84 & 0.000 & Fatty acid metabolism, oxidative injury \\
\hline & 3 & 1.20 & 258.1108 & $\mathrm{C}_{8} \mathrm{H}_{21} \mathrm{NO}_{6} \mathrm{P}$ & Glycerophosphocholine $^{c}$ & 25.39 & 0.000 & Glycerophospholipid metabolism \\
\hline & 4 & 1.73 & 204.1235 & $\mathrm{C}_{9} \mathrm{H}_{17} \mathrm{NO}_{4}$ & Acetylcarnitine $e^{c}$ & 0.819 & 0.006 & Fatty acid metabolism, oxidative injury \\
\hline & 5 & 3.06 & 166.0869 & $\mathrm{C}_{9} \mathrm{H}_{11} \mathrm{NO}_{2}$ & L-Phenylalanine $\mathrm{b}^{\mathrm{b}}$ & 0.81 & 0.005 & Phenylalanine metabolism \\
\hline & 6 & 5.07 & 180.0661 & $\mathrm{C}_{9} \mathrm{H}_{9} \mathrm{NO}_{3}$ & Hippuric acid ${ }^{\mathrm{b}}$ & 25.27 & 0.007 & Phenylalanine metabolism \\
\hline & 7 & 5.30 & 194.0820 & $\mathrm{C}_{10} \mathrm{H}_{11} \mathrm{NO}_{3}$ & Phenylacetylglycine ${ }^{c}$ & -0.93 & 0.004 & Phenylalanine metabolism \\
\hline & 8 & 10.13 & 357.2796 & $\mathrm{C}_{24} \mathrm{H}_{36} \mathrm{O}_{2}$ & DHA ethyl ester ${ }^{c}$ & 3.19 & 0.002 & $\begin{array}{l}\text { Alpha linolenic acid and linoleic acid } \\
\text { metabolism }\end{array}$ \\
\hline & 9 & 14.89 & 400.3427 & $\mathrm{C}_{23} \mathrm{H}_{45} \mathrm{NO}_{4}$ & Palmitoyl-L-carnitine $^{c}$ & 0.57 & 0.003 & Fatty acid metabolism \\
\hline & 10 & 16.58 & 305.2481 & $\mathrm{C}_{20} \mathrm{H}_{32} \mathrm{O}_{2}$ & Arachidonic $\mathrm{acid}^{\mathrm{b}}$ & 0.94 & 0.002 & Arachidonic acid metabolism \\
\hline & 11 & 16.79 & 271.2637 & $\mathrm{C}_{17} \mathrm{H}_{34} \mathrm{O}_{2}$ & Palmitic acid methyl ester ${ }^{c}$ & 4.09 & 0.000 & Fatty acid metabolism \\
\hline \multirow{7}{*}{ ESI(-) } & 12 & 1.24 & 157.0361 & $\mathrm{C}_{4} \mathrm{H}_{6} \mathrm{~N}_{4} \mathrm{O}_{3}$ & Allantoin $^{\mathrm{b}}$ & 2.13 & 0.000 & Purine metabolism, oxidative injury \\
\hline & 13 & 1.74 & 167.0207 & $\mathrm{C}_{5} \mathrm{H}_{4} \mathrm{~N}_{4} \mathrm{O}_{3}$ & Uric acid ${ }^{\mathrm{b}}$ & -1.16 & 0.004 & Purine metabolism, oxidative injury \\
\hline & 14 & 2.19 & 219.0775 & $\mathrm{C}_{11} \mathrm{H}_{12} \mathrm{~N}_{2} \mathrm{O}_{3}$ & 5-Hydroxy tryptophan ${ }^{\mathrm{b}}$ & 0.99 & 0.008 & Tryptophan metabolism \\
\hline & 15 & 4.51 & 203.0831 & $\mathrm{C}_{11} \mathrm{H}_{12} \mathrm{~N}_{2} \mathrm{O}_{2}$ & L-Tryptophan ${ }^{\mathrm{b}}$ & 1.61 & 0.003 & Tryptophan metabolism \\
\hline & 16 & 8.34 & 212.0025 & $\mathrm{C}_{8} \mathrm{H}_{7} \mathrm{NO}_{4} \mathrm{~S}$ & Indoxyl sulfate $e^{c}$ & 1.27 & 0.003 & Tryptophan metabolism \\
\hline & 17 & 6.21 & 464.3024 & $\mathrm{C}_{26} \mathrm{H}_{43} \mathrm{NO}_{6}$ & Glycocholic acid ${ }^{c}$ & -0.38 & 0.022 & Bile acid biosynthesis \\
\hline & 18 & 10.14 & 391.2855 & $\mathrm{C}_{24} \mathrm{H}_{40} \mathrm{O}_{4}$ & Deoxycholic acid ${ }^{c}$ & -0.44 & 0.046 & Bile acid biosynthesis \\
\hline
\end{tabular}

a Fold changes (calculated as $\log _{2}$ (average peak intensity of model group/average peak intensity of normal control group)) and $P$ value compared with normal control group on day 22 .

${ }^{\mathrm{b}}$ Metabolites validated with standards.

${ }^{\mathrm{c}}$ Metabolites putatively annotated.

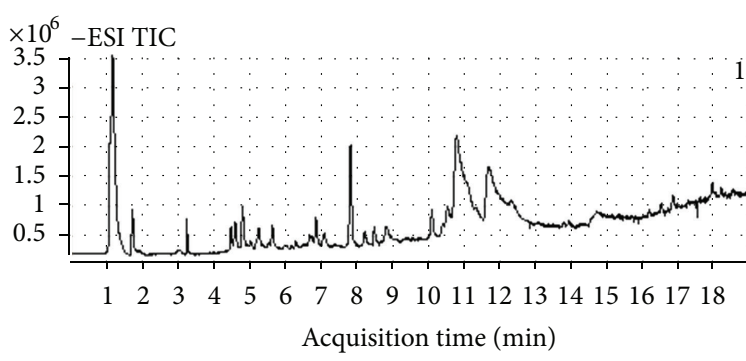

(a)

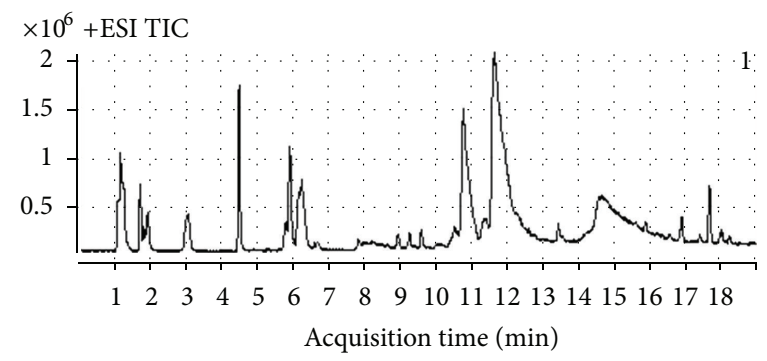

(b)

FIGURE 2: Representative base peak intensity chromatogram of the rat plasma obtained in ESI negative mode (a) and ESI positive mode (b) based on LC-Q-TOF-MS.

of urine metabolomics; the results obtained here were complementary to our earlier study [23].

In summary, 18 potential biomarkers were identified. These markers are mainly associated with glycerophospholipid metabolism, fatty acid metabolism, tryptophan metabolism, linoleic acid metabolism, phenylalanine metabolism, purine metabolism, arachidonic acid metabolism, and bile acid biosynthesis and reveal RA regulating network in vivo.

3.5. Metabolomic Analysis of HLJDT and Its Treatment Components. PCA, an unsupervised pattern recognition method, was used to observe trends in mean metabolite pattern changes across various time points (Figure 6). PLS-DA, a supervised pattern recognition method, was used to display the metabolic state of CIA rats on days 8, 15, and 22 (Figure 7). The location marked with an arrow in Figure 6 indicates the trend in mean metabolite pattern changes. On day 8 , each group's metabolic state had changed from its initial position (day 0). This change indicates that RA had disrupted endogenous substance metabolism and had significantly altered the metabolic fingerprints of the plasma compared to its normal state. From day 8 to day 22, the direction of the trajectory gradually moved towards the initial space. The trajectory then returned to the initial space, indicating a recovery from the disrupted metabolic state. Compared to the model control group, the three drug treatment groups showed better recovery performance from the CIA-induced metabolic state. This result can be observed by comparing the dynamic 


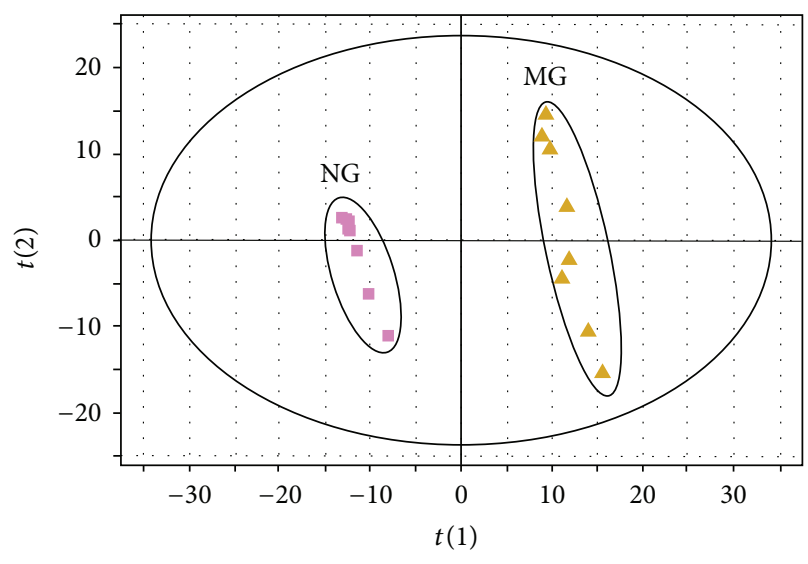

(a)

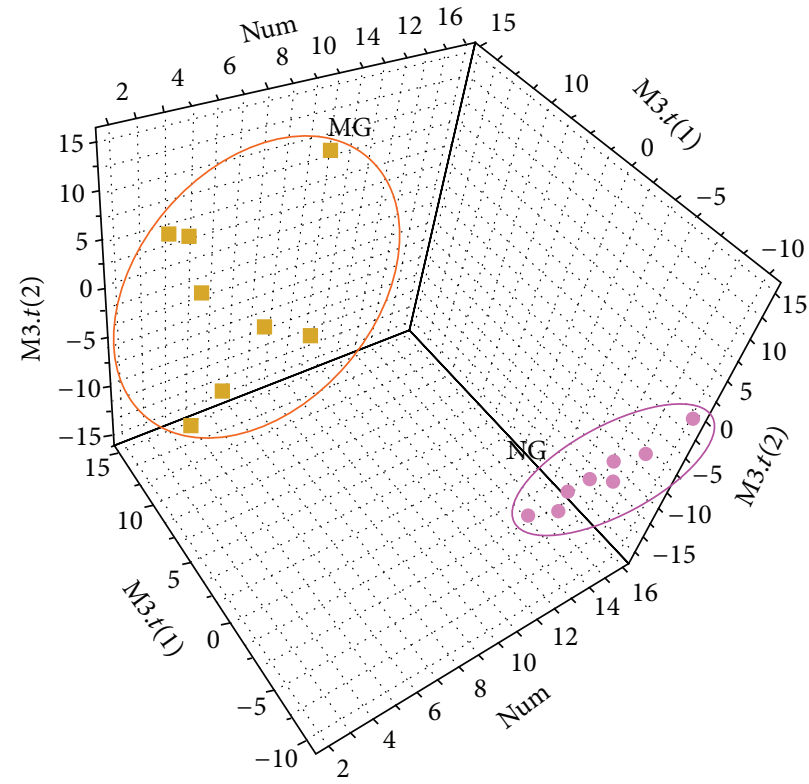

(b)

FIGURE 3: Results of multiple pattern recognition of plasma biomarkers between normal control group and model group on day 22. PLS-DA score plot $\left(R^{2} X=0.253, R^{2} Y=0.997, Q_{2}=0.875, n=8\right)$ of NG and MG. NG, normal control group; MG, model group.

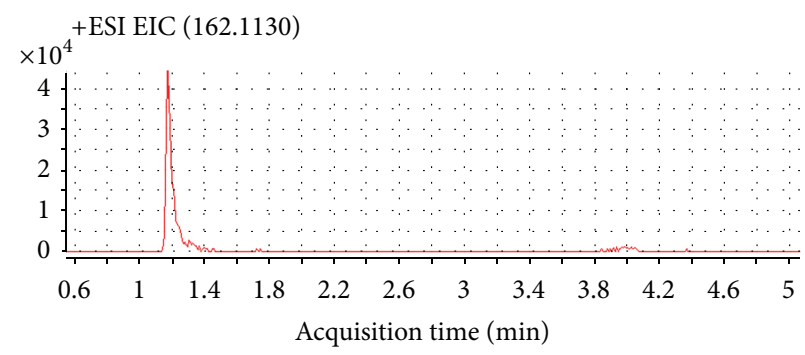

(a)

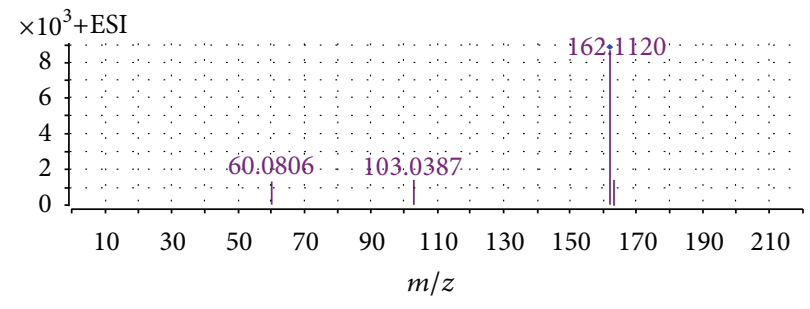

(b)

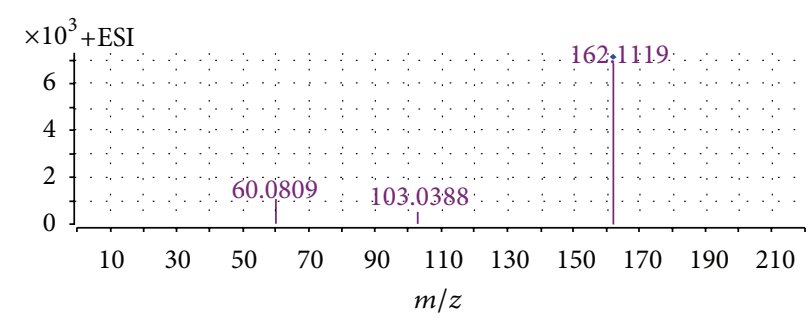

(c)

FIGURE 4: Identification of a selected biomarker ( $\mathrm{m} / z$ 162.1129). (a) Extracted ion chromatogram (EIC) of $\mathrm{m} / z 162.113\left(t_{R}=1.19 \mathrm{~min}\right)$; (b) MS/MS spectrum of the ion; (c) MS/MS spectrum of a commercial standard carnitine. The collision energy was $10 \mathrm{~V}$.

trajectories in Figures 6 and 7(c). While the dexamethasone group had the advantage of rapid treatment, the toxic side effects of long-term dexamethasone administration led to a metabolic state that deviated from normal rats on day 22. Furthermore, HLJDT and its components resulted in better recovery performance from the CIA-induced metabolic state than dexamethasone on day 22 (Figure $7(\mathrm{c})$ ).
Nine metabolites were reversed by HLJDT, and 7 were reversed by its components (Table 3 ). This result indicates that the component group could largely replace the effects of the complete formula. The metabolites that were reversed are primarily involved in phenylalanine metabolism, glycerophospholipid metabolism, fatty acid metabolism, and arachidonic acid metabolism, which indicate that the 


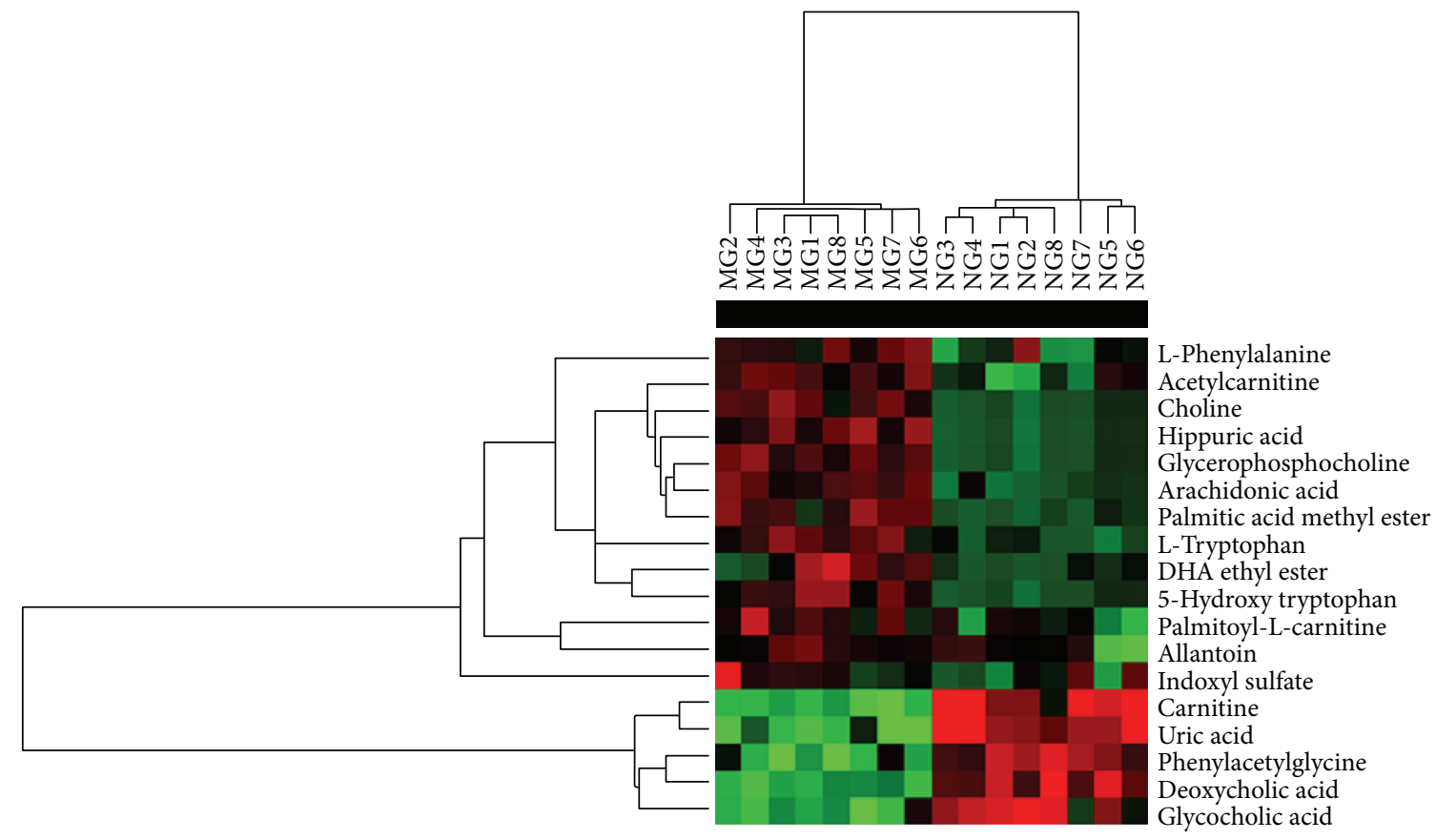

FIGURE 5: All the potential biomarkers in response to RA detected by cluster analysis. The columns show the expression levels and each row represents a biomarker. The red color indicates upregulated biomarkers compared with normal control group, while the green color represents downregulated biomarkers compared with normal control group. NG, normal control group; MG, model group; $n=8$.

TABLE 3: Summary of potential biomarkers in HLJDT and its components' groups on day 22.

\begin{tabular}{lcccc}
\hline \multirow{2}{*}{ Biomarkers } & \multicolumn{2}{c}{ HLJDT } & \multicolumn{2}{c}{ Components of HLJDT } \\
& Fold $^{\mathrm{a}}$ & $P$ value $^{\mathrm{a}}$ & Fold $^{\mathrm{b}}$ & $P$ value $^{\mathrm{b}}$ \\
\hline Choline & -20.24 & 0.041 & - & - \\
Carnitine & 0.40 & 0.000 & 0.18 & 0.003 \\
Glycerophosphocholine & -19.87 & 0.001 & -16.79 & 0.009 \\
Acetylcarnitine & -0.76 & 0.003 & -0.15 & 0.002 \\
L-Phenylalanine & -0.28 & 0.012 & -0.19 & 0.043 \\
Hippuric acid & -20.17 & 0.004 & -18.87 & 0.006 \\
Palmitoyl-L-carnitine & -0.56 & 0.002 & -0.01 & 0.022 \\
Allantoin & -0.12 & 0.018 & - & - \\
Arachidonic acid & -0.24 & 0.002 & -0.14 & 0.003 \\
\hline
\end{tabular}

${ }^{\mathrm{a}}$ Fold changes calculated as $\log _{2}$ (average peak intensity of HLJDT group/average peak intensity of model group) and $P$ value compared with model group on day 22 .

${ }^{\mathrm{b}}$ Fold changes calculated as $\log _{2}$ (average peak intensity of components of HLJDT group/average peak intensity of model group) and $P$ value compared with model group on day 22 .

effectiveness of HLJDT and its components as an RA treatment partially depends on restoring imbalances related to oxidative injury and the arachidonic acid pathway. HLJDT was reported to have protective and therapeutic effects on peripheral inflammation and hepatotoxin-induced liver injuries [41, 42]. Rhizoma coptidis and Radix scutellariae were responsible for the suppressive effect of HLJDT on eicosanoid generation. Some of their pure components, including baicalein, baicalin, wogonoside, wogonin, coptisine, and magnoflorine, were also shown to inhibit eicosanoid generation in rat macrophages via the arachidonic acid cascade [21].

The results from a lipid peroxide assay, antioxidant enzyme activity assays, and metabolomic analysis demonstrate that HLJDT and its components have extensive effects in RA treatment by regulating the pathway disruptions associated with oxidative injury and arachidonic acid metabolism.

\section{Conclusions}

In this study, metabolomic analysis with LC-Q-TOF-MS was used to profile RA-related metabolic changes in the plasma and to investigate the interventional mechanisms of HLJDT and its components. After multiple levels of statistical analysis, 18 significant biomarkers (11 metabolites detected in the positive mode and 7 metabolites detected in the negative mode) were identified. These biomarkers are primarily involved in glycerophospholipid metabolism, fatty acid metabolism, tryptophan metabolism, linoleic acid metabolism, phenylalanine metabolism, purine metabolism, arachidonic acid metabolism, and bile acid biosynthesis. Potential biomarkers-related glycerophospholipid metabolism and fatty acid metabolism, namely, carnitine, acetylcarnitine, allantoin, uric acid, choline, and glycerophosphocholine, appear to have diagnostic and/or prognostic values for RA and require further investigation in clinical studies. Using the potential biomarkers identified in this study as a screening index, we hypothesize that HLJDT 


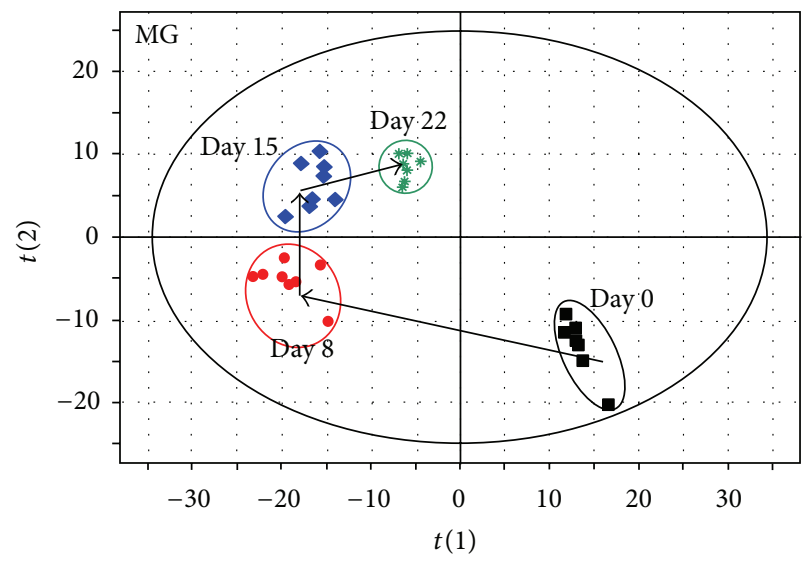

(a)

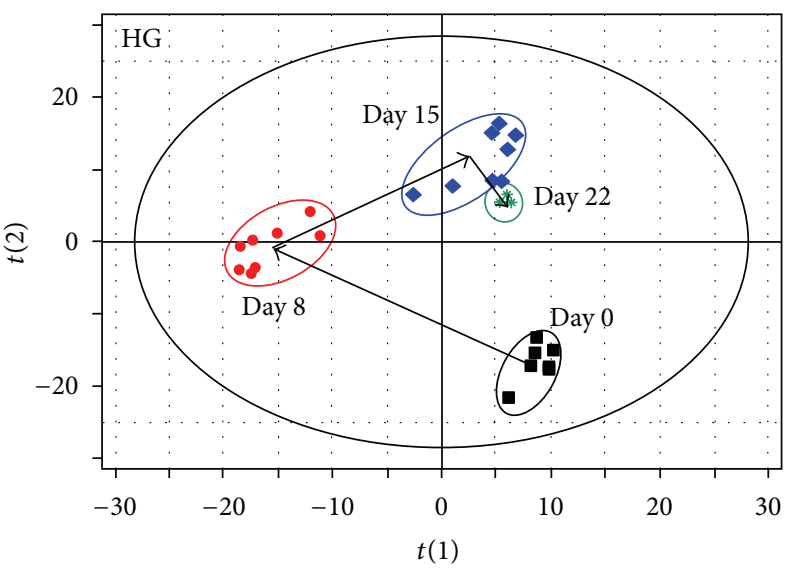

(c)

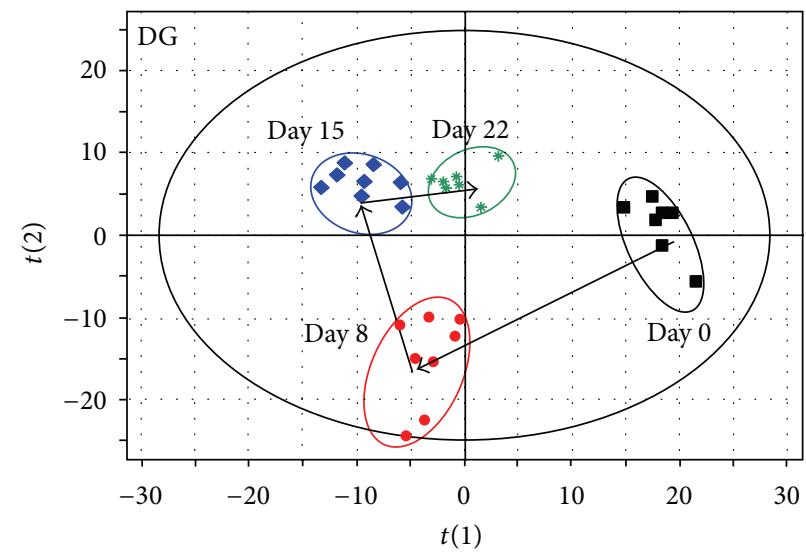

(b)

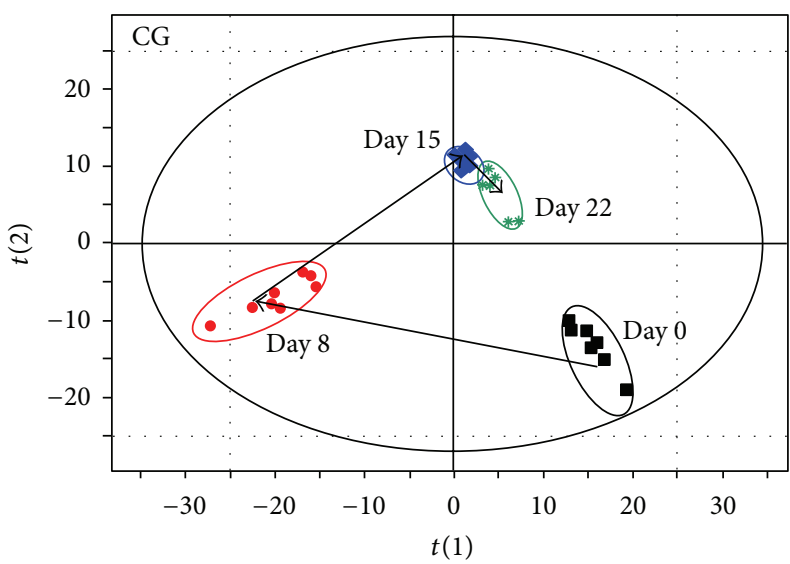

(d)

FIGURE 6: Dynamic PCA scores' plots of plasma metabolites impacted by different groups from day 0 to 22. (a) HLJDT group, (b) dexamethasone group, (c) components group, and (d) model group. HG, HLJDT group; DG, dexamethasone group; CG, components group; MG, model group.

and its components can limit the pathological process of RA by partially reversing metabolite levels and regulating pathway disruptions. The metabolomic results presented here provide a systemic view of the development and progression of RA as well as a theoretical basis for the prevention or treatment of RA.

\section{Abbreviations}

RA:

Rheumatoid arthritis

CIA:

Collagen-induced arthritis

LC-Q-TOF-MS: Liquid chromatography quadrupole time-of-flight mass spectrometry

HLJDT: $\quad$ Huang-Lian-Jie-Du-Tang formula

NG:

MG: Normal group

DG: Model group

HG: Dexamethasone group

CG:

TCM: HLJDT group Components group

PCA:
PLS-DA: Partial least squares discriminant analysis

GSH-Px: Glutathione peroxidase

SOD: Superoxide dismutase

MDA: Malondialdehyde

TBA: Thiobarbituric acid.

\section{Conflict of Interest}

The authors declare that they have no conflict of interests.

\section{Authors' Contribution}

R. Yue and L. Zhao contributed equally to this paper.

\section{Acknowledgments}

Thid work was supported by NSFC $(81102865,81230090$, and 30725045), partially supported by Global Research Network for Medicinal Plants (GRNMP), Shanghai Leading Academic Discipline Project (B906), FP7-PEOPLE-IRSES-2008 


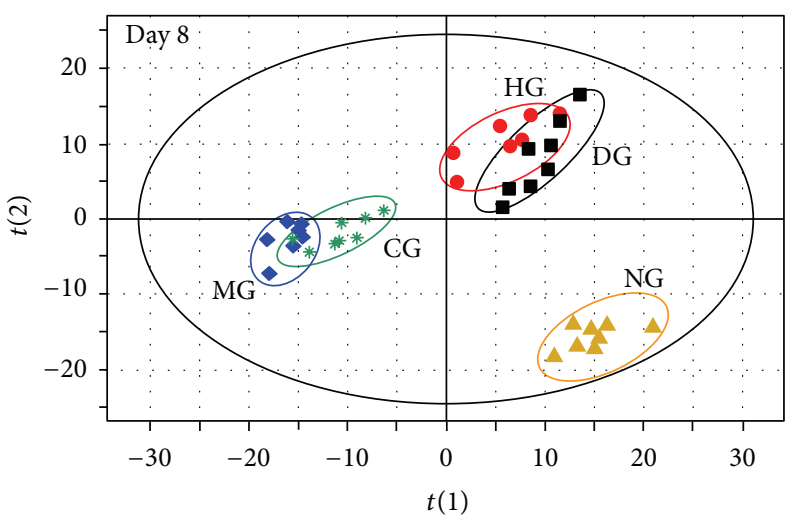

(a)

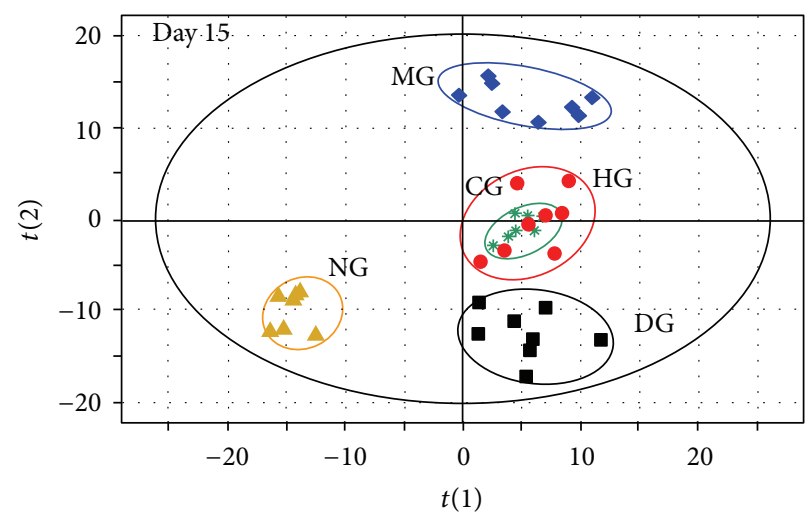

(b)

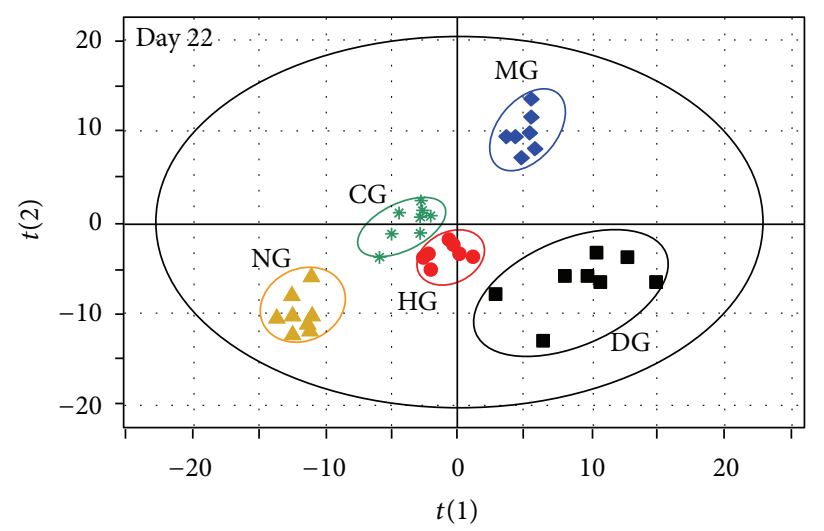

(c)

Figure 7: Comparison of PLS-DA scores plots of rat plasma data of different groups on days 8, 15, and 22. (a) Day 8 ( $Q^{2} Y_{\text {(cum) }}=0.777$, $\left.R^{2} X_{(\mathrm{cum})}=0.428, R^{2} Y_{(\mathrm{cum})}=0.98\right)$. (b) Day $15\left(Q^{2} Y_{(\mathrm{cum})}=0.796, R^{2} X_{(\mathrm{cum})}=0.41, R^{2} Y_{(\mathrm{cum})}=0.985\right) .(\mathrm{c}) \mathrm{Day} 22\left(Q^{2} Y_{(\mathrm{cum})}=0.804\right.$, $\left.R^{2} X_{\text {(cum) }}=0.439, R^{2} Y_{\text {(cum) }}=0.993\right)$. NG, normal control group; MG, model group; DG, dexamethasone group; HG, HLJDT group; CG, components group.

(TCMCANCER Project 230232), Key Laboratory of Drug Research for Special Environments, PLA, Shanghai Engineering Research Center for the Preparation of Bioactive Natural Products (10DZ2251300), the Scientific Foundation of Shanghai China (09DZ1975700, 09DZ1971500, and 10DZ1971700), National Major Project of China (2011ZX09307-002-03), and the Twelfth Five-Year National Science and Technology Support Program (2012BAI29B06).

\section{References}

[1] F. C. Arnett, S. M. Edworthy, D. A. Bloch et al., "The American Rheumatism Association 1987 revised criteria for the classification of rheumatoid arthritis," Arthritis and Rheumatism, vol. 31, no. 3, pp. 315-324, 1988.

[2] D. L. Scott, F. Wolfe, and T. W. J. Huizinga, "Rheumatoid arthritis," The Lancet, vol. 376, no. 9746, pp. 1094-1108, 2010.

[3] M. A. van Maanen, M. C. Lebre, T. van der Poll et al., "Stimulation of nicotinic acetylcholine receptors attenuates collageninduced arthritis in mice," Arthritis and Rheumatism, vol. 60, no. 1, pp. 114-122, 2009.
[4] W. Q. Lai, A. W. Irwan, H. H. Goh, A. J. Melendez, I. B. McInnes, and B. P. Leung, "Distinct roles of sphingosine kinase 1 and 2 in murine collagen-induced arthritis," Journal of Immunology, vol. 183, no. 3, pp. 2097-2103, 2009.

[5] H. Kelchtermans, L. Geboes, T. Mitera, D. Huskens, G. Leclercq, and P. Matthys, "Activated CD4+CD25+ regulatory T cells inhibit osteoclastogenesis and collagen-induced arthritis," Annals of the Rheumatic Diseases, vol. 68, no. 5, pp. 744-750, 2009.

[6] L. G. M. van Baarsen, C. A. Wijbrandts, T. C. G. Timmer, T. C. T. M. van der Pouw Kraan, P. P. Tak, and C. L. Verweij, "Synovial tissue heterogeneity in rheumatoid arthritis in relation to disease activity and biomarkers in peripheral blood," Arthritis and Rheumatism, vol. 62, no. 6, pp. 1602-1607, 2010.

[7] V. C. Willis, A. M. Gizinski, N. K. Banda et al., "N- $\alpha-$ benzoyl-N5-(2-chloro-1-iminoethyl)-L-ornithine amide, a protein arginine deiminase inhibitor, reduces the severity of murine collagen-induced arthritis," Journal of Immunology, vol. 186, no. 7, pp. 4396-4404, 2011.

[8] A. Augello, R. Tasso, S. M. Negrini, R. Cancedda, and G. Pennesi, "Cell therapy using allogeneic bone marrow mesenchymal 
stem cells prevents tissue damage in collagen-induced arthritis," Arthritis and Rheumatism, vol. 56, no. 4, pp. 1175-1186, 2007.

[9] C. Salliot and D. van der Heijde, "Long-term safety of methotrexate monotherapy in patients with rheumatoid arthritis: a systematic literature research," Annals of the Rheumatic Diseases, vol. 68, no. 7, pp. 1100-1104, 2009.

[10] N. Alcorn, S. Saunders, and R. Madhok, "Benefit-risk assessment of leflunomide: an appraisal of leflunomide in rheumatoid arthritis 10 years after licensing," Drug Safety, vol. 32, no. 12, pp. 1123-1134, 2009.

[11] D. Schaffer, T. Florin, C. Eagle et al., "Risk of serious NSAIDrelated gastrointestinal events during long-term exposure: a systematic review," Medical Journal of Australia, vol. 185, no. 9, pp. 501-506, 2006.

[12] P. A. Scott, G. H. Kingsley, C. M. Smith, E. H. Choy, and D. L. Scott, "Non-steroidal anti-inflammatory drugs and myocardial infarctions: comparative systematic review of evidence from observational studies and randomised controlled trials," Annals of the Rheumatic Diseases, vol. 66, no. 10, pp. 1296-1304, 2007.

[13] C. Gieger, L. Geistlinger, E. Altmaier et al., "Genetics meets metabolomics: a genome-wide association study of metabolite profiles in human serum," PLoS Genetics, vol. 4, no. 11, Article ID e1000282, 2008.

[14] A. M. Weljie, R. Dowlatabadi, B. J. Miller, H. J. Vogel, and F. R. Jirik, "An inflammatory arthritis-associated metabolite biomarker pattern revealed by $1 \mathrm{H}$ NMR spectroscopy," Journal of Proteome Research, vol. 6, no. 9, pp. 3456-3464, 2007.

[15] M. B. Lauridsen, H. Bliddal, R. Christensen et al., "1H NMR spectroscopy-based interventional metabolic phenotyping: a cohort study of rheumatoid arthritis patients," Journal of Proteome Research, vol. 9, no. 9, pp. 4545-4553, 2010.

[16] X. Li, S. Yang, Y. Qiu et al., "Urinary metabolomics as a potentially novel diagnostic and stratification tool for knee osteoarthritis," Metabolomics, vol. 6, no. 1, pp. 109-118, 2010.

[17] G. Theodoridis, H. G. Gika, and I. D. Wilson, "LC-MS-based methodology for global metabolite profiling in metabonomics/ metabolomics," Trends in Analytical Chemistry, vol. 27, no. 3, pp. 251-260, 2008.

[18] A. Nordström, G. O’Maille, C. Qin, and G. Siuzdak, "Nonlinear data alignment for UPLC-MS and HPLC-MS based metabolomics: quantitative analysis of endogenous and exogenous metabolites in human serum," Analytical Chemistry, vol. 78, no. 10, pp. 3289-3295, 2006.

[19] S. Dou, L. Liu, P. Jiang, W. Zhang, and R. Liu, "LC-DAD and LC-ESI-MS chromatographic fingerprinting and quantitative analysis for evaluation of the quality of Huang-Lian-Jie-DuTang," Chromatographia, vol. 69, no. 7-8, pp. 659-664, 2009.

[20] Y. Hu, P. Jiang, S. Wang et al., "Plasma pharmacochemistry based approach to screening potential bioactive components in Huang-Lian-Jie-Du-Tang using high performance liquid chromatography coupled with mass spectrometric detection," Journal of Ethnopharmacology, vol. 141, no. 2, pp. 728-735, 2012.

[21] H. Zeng, S. Dou, J. Zhao et al., "The inhibitory activities of the components of Huang-Lian-Jie-Du-Tang (HLJDT) on eicosanoid generation via lipoxygenase pathway," Journal of Ethnopharmacology, vol. 135, no. 2, pp. 561-568, 2011.

[22] H. Zeng, X. Liu, S. Dou et al., "Huang-Lian-Jie-Du-Tang exerts anti-inflammatory effects in rats through inhibition of nitric oxide production and eicosanoid biosynthesis via the lipoxygenase pathway," Journal of Pharmacy and Pharmacology, vol. 61, no. 12, pp. 1699-1707, 2009.
[23] R. Yue, L. Zhao, Y. Hu et al., "Rapid-resolution liquid chromatography TOF-MS for urine metabolomic analysis of collagen-induced arthritis in rats and its applications," Journal of Ethnopharmacology, vol. 145, no. 2, pp. 465-475, 2013.

[24] C. A. Hitchon and H. S. El-Gabalawy, "Oxidation in rheumatoid arthritis," Arthritis Research and Therapy, vol. 6, no. 6, pp. 265278, 2004.

[25] G. Baskol, H. Demir, M. Baskol et al., "Investigation of protein oxidation and lipid peroxidation in patients with rheumatoid arthritis," Cell Biochemistry and Function, vol. 24, no. 4, pp. 307311, 2006.

[26] H. R. Griffiths, "Is the generation of neo-antigenic determinants by free radicals central to the development of autoimmune rheumatoid disease?" Autoimmunity Reviews, vol. 7, no. 7, pp. 544-549, 2008.

[27] K. Hadjigogos, "The role of free radicals in the pathogenesis of rheumatoid arthritis," Panminerva Medica, vol. 45, no. 1, pp. 713, 2003.

[28] L. Gail Darlington and T. W. Stone, "Antioxidants and fatty acids in the amelioration of rheumatoid arthritis and related disorders," British Journal of Nutrition, vol. 85, no. 3, pp. 251269, 2001.

[29] E. A. Ostrakhovitch and I. B. Afanas'ev, "Oxidative stress in rheumatoid arthritis leukocytes: suppression by rutin and other antioxidants and chelators," Biochemical Pharmacology, vol. 62, no. 6, pp. 743-746, 2001.

[30] B. N. Ames, R. Cathcart, E. Schwiers, and P. Hochstein, "Uric acid provides an antioxidant defense in humans against oxidant- and radical-caused aging and cancer: a hypothesis," Proceedings of the National Academy of Sciences of the United States of America, vol. 78, no. 11, pp. 6858-6862, 1981.

[31] M. Grootveld and B. Halliwell, "Measurement of allantoin and uric acid in human body fluids. A potential index of free-radical reactions in vivo?" Biochemical Journal, vol. 243, no. 3, pp. 803808, 1987.

[32] G. K. Glantzounis, E. C. Tsimoyiannis, A. M. Kappas, and D. A. Galaris, "Uric acid and oxidative stress," Current Pharmaceutical Design, vol. 11, no. 32, pp. 4145-4151, 2005.

[33] A. M. Cohen, R. E. Aberdroth, and P. Hochstein, "Inhibition of free radical-induced DNA damage by uric acid," FEBS Letters, vol. 174, no. 1, pp. 147-150, 1984.

[34] K. J. A. Davies, A. Sevanian, S. F. Muakkassah-Kelly, and P. Hochstein, "Uric acid-iron ion complexes. A new aspect of the antioxidant functions of uric acid," Biochemical Journal, vol. 235, no. 3, pp. 747-754, 1986.

[35] N. Tastekin, N. Aydogdu, D. Dokmeci et al., "Protective effects of l-carnitine and alpha-lipoic acid in rats with adjuvant arthritis," Pharmacological Research, vol. 56, no. 4, pp. 303-310, 2007.

[36] C. R. Bruce, A. J. Hoy, N. Turner et al., "Overexpression of carnitine palmitoyltransferase-1 in skeletal muscle is sufficient to enhance fatty acid oxidation and improve high-fat dietinduced insulin resistance," Diabetes, vol. 58, no. 3, pp. 550-558, 2009.

[37] F. Le Borgne, A. Ben Mohamed, M. Logerot, E. Garnier, and J. Demarquoy, "Changes in carnitine octanoyltransferase activity induce alteration in fatty acid metabolism," Biochemical and Biophysical Research Communications, vol. 409, no. 4, pp. 699704, 2011. 
[38] A. Kllzllltunc, S. Cogalgil, and L. Cerrahoglu, "Carnitine and antioxidants levels in patients with rheumatoid arthritis," Scandinavian Journal of Rheumatology, vol. 27, no. 6, pp. 441-445, 1998.

[39] S. Krähenbühl, B. Willer, P. Brühlmann, H. Hoppeler, and G. Stucki, "Carnitine homeostasis in patients with rheumatoid arthritis," Clinica Chimica Acta, vol. 279, no. 1-2, pp. 35-45, 1999.

[40] A. Bruusgaard and R. B. Andersen, "Effect of an intravenously administered bile acid (chenodeoxycholic acid) on rheumatoid arthritis," Scandinavian Journal of Rheumatology, vol. 4, no. 3, pp. 169-173, 1975.

[41] Y. Dai, K. Miki, T. Fukuoka et al., 'Suppression of neuropeptides' mRNA expression by herbal medicines in a rat model of peripheral inflammation," Life Sciences, vol. 66, no. 1, pp. 19-29, 1999.

[42] S. C. Lin, C. C. Lin, F. J. Lu, Y. H. Lin, and C. H. Chen, "Protective and therapeutic effects of huanglian-jie-du-tang on hepatotoxin-induced liver injuries," American Journal of Chinese Medicine, vol. 24, no. 3-4, pp. 219-229, 1996. 


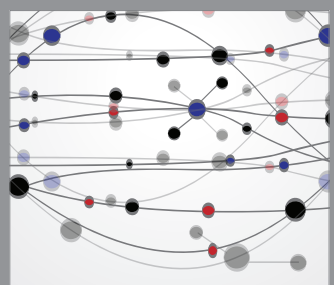

The Scientific World Journal
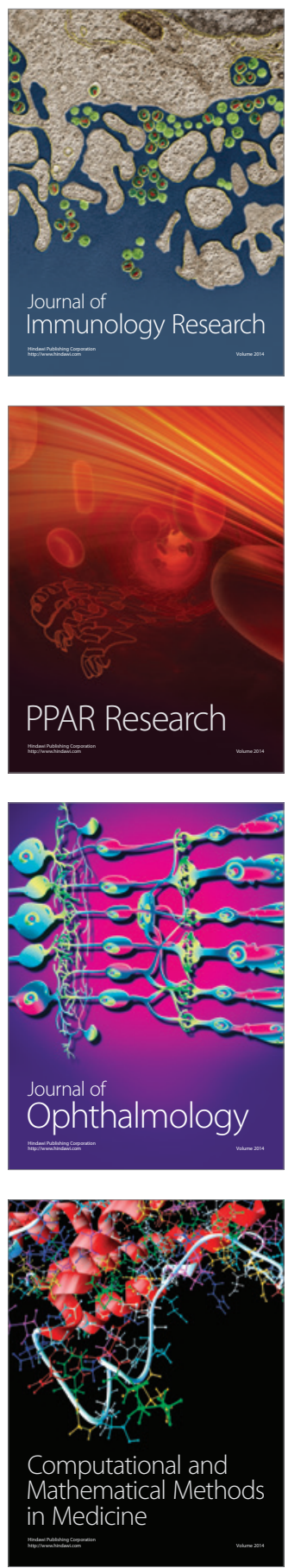

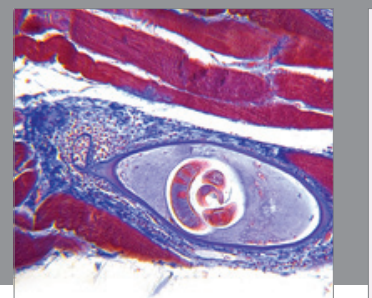

Gastroenterology

Research and Practice
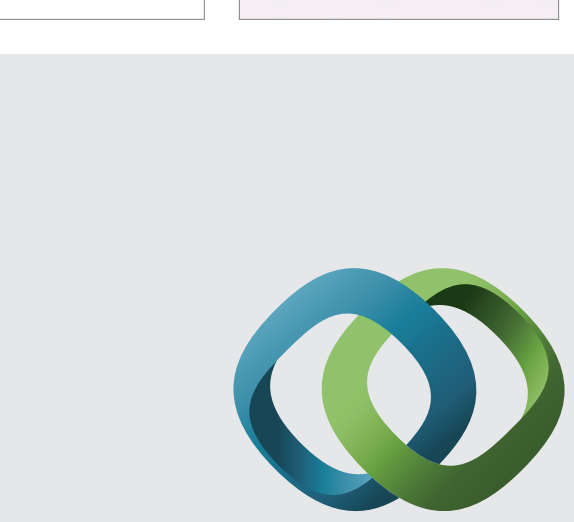

\section{Hindawi}

Submit your manuscripts at

http://www.hindawi.com
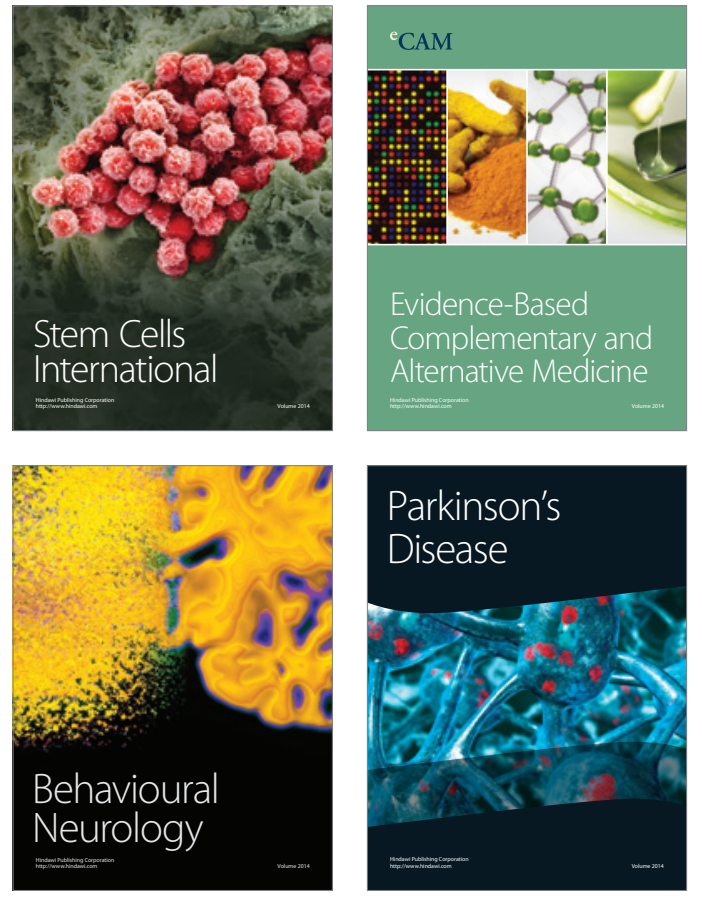
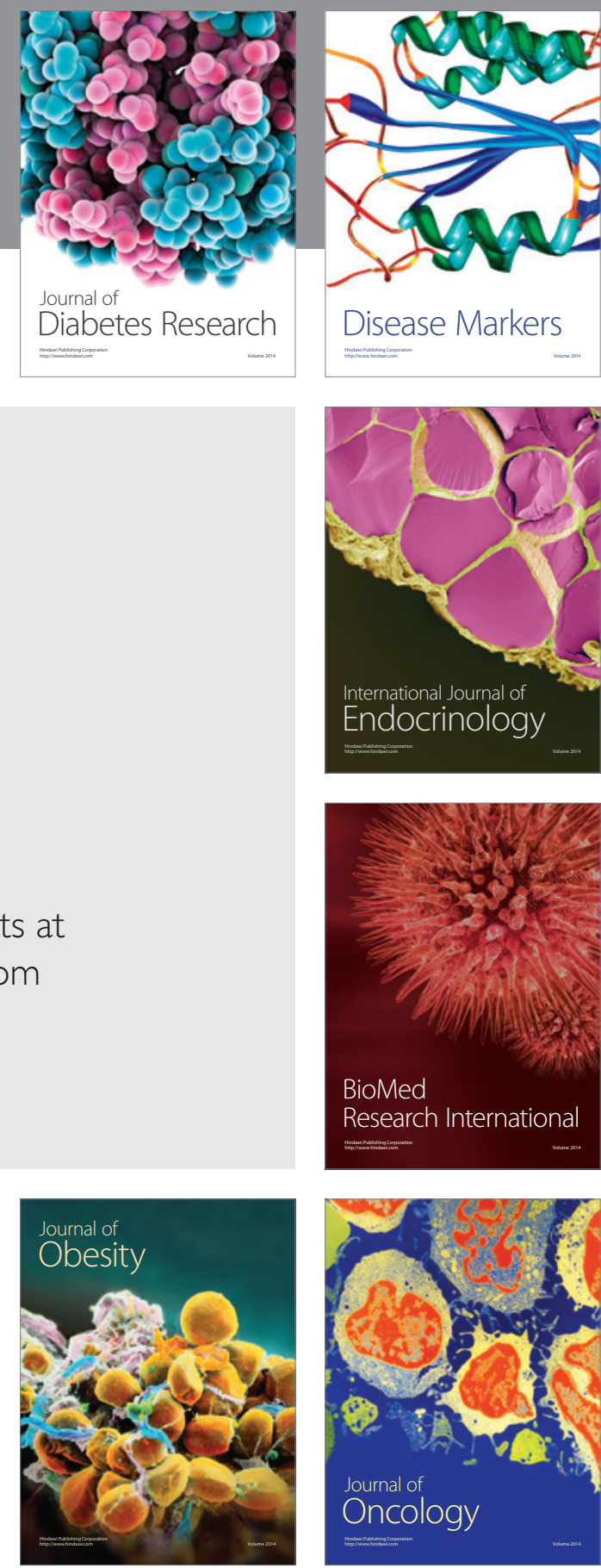

Disease Markers
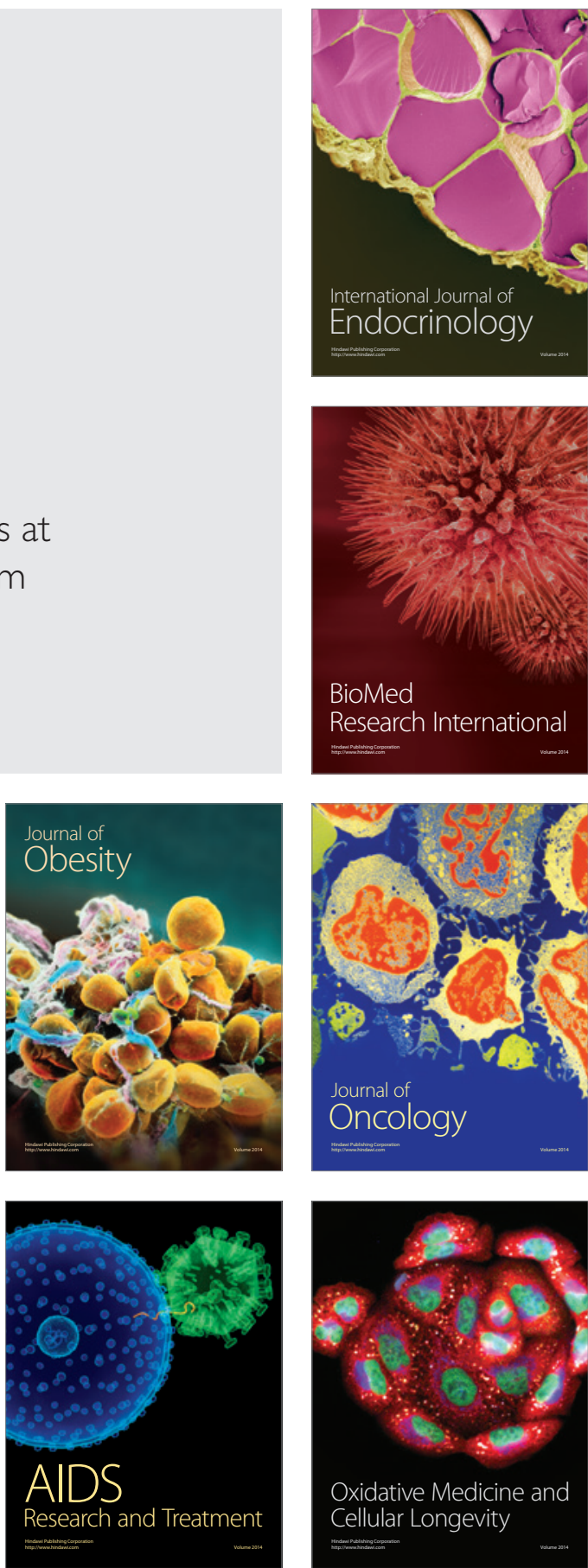